\title{
Association of Cytology, Microbiology and Histopathology of Cytobrush, Uterine Lavage and Endometrial Biopsy Samples from Mares with Endometritis
}

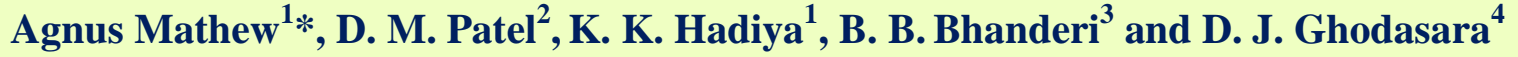 \\ ${ }^{1}$ Department of Veterinary Gynaecology \& Obstetrics, ${ }^{2}$ Dept. of Veterinary Clinical Complex, \\ ${ }^{3}$ Department of Veterinary Microbiology, ${ }^{4}$ Department of Veterinary Pathology, \\ College of Veterinary Science and AH, Anand Agril University, Anand-388 001, India
}

*Corresponding author

\section{A B S T R A C T}

\begin{tabular}{|l|}
\hline K e y w o r d s \\
Endometritis, Mare, \\
Cytobrush, \\
Endometrial biopsy, \\
Low volume uterine \\
lavage, \\
Bacteriology, \\
Cytology, \\
Histopathology. \\
\hline Article Info \\
\hline $\begin{array}{l}\text { Accepted: } \\
\text { 15 May } 2020 \\
\text { Available Online: } \\
\text { 10 June } 2020\end{array}$ \\
\hline
\end{tabular}

The samples collected for comparison of the three techniques, viz., cytobrush (CB), uterine lavage (LVL) and endometrial biopsy (EB) from 10 mares with endometritis were evaluated by cytological, bacteriological and histological methods. A total of $6(60 \%)$ mares had positive histopathology for endometritis. The results of cytological examinations were compared for the presence of infiltration by PMNs of the luminal epithelium and stratum compactum as histological examination of the endometrium is generally used as the reference standard for diagnosis of endometritis. Positive growth from EB was obtained in $7(70 \%)$ of all mares, from $\mathrm{CB}$ in $6(60 \%)$ and from LVL in $8(80 \%)$ mares. No statistical differences in the number of positive cultures were obtained between different techniques. In 3 of 10 (30\%) cytological smears obtained from EB and CB and in 4 of 10 (40\%) cytological smears obtained from LVL, the percentage of PMNs was $\geq 1$. This difference was not significant. In EB, CB and LVL, all the cytologically positive mares also had positive cultures. Whereas, $30 \%$ positive cultures from $\mathrm{CB}$ and $40 \%$ positive cultures from EB and LVL had negative cytology. The most common isolated bacteria were $\beta$-hemolytic Streptococci and E. coli either alone (30 \%) or in combination in all three techniques. There were no differences in the number of positive cultures between CB, EB and LVL or in positive cytology findings between the three methods. Isolation of $\beta$-hemolytic Streptococci from positive cytology was indicative of their pathogenicity. The findings however warrant further study on larger sample size on mares with clinical and subclinical endometritis.

\section{Introduction}

Equine endometritis has a significant economic impact as it affects estrous cycles and creates infertility (LeBlanc, 2010). It occurs due to foreign molecules when introduced into the reproductive tract specifically at mating (Riddle et al., 2007). The inflammatory response is characterized by an influx of polymorphonuclear neutrophils (PMNs) in the luminal fluid, endometrial edema, vaginal discharge and cervicitis. Bacteria such as Pseudomonas aeruginosa and some yeast and fungi are known to form biofilms (Ferris et al., 2014), resulting in persistent, chronic infections, yet these mares are often negative on culture and cytology (Beehan et al., 2015). Endometrial cytology of samples obtained by 
different techniques is a readily available diagnostic test to identify mares with endometritis, although false negatives can be increased with focal/localized sampling (LeBlanc et al., 2007). However, uterine biopsy is often considered as the "gold standard" (LeBlanc and Causey, 2009) and bacteriological examination provides an indirect test for the diagnosis of endometritis in mares. The literature on these aspects in Indian context is meager in equines. It is hypothesized that combining diagnostic methods into an endometritis checklist would increase confidence in correctly diagnosing mares with endometritis. Hence, this investigation was undertaken to study the association of cytological, bacteriological and histological evaluation of uterine samples collected by cytobrush, endometrial biopsy and low volume lavage from infertile mares.

\section{Materials and Methods}

\section{Experimental animals and endometrial sample collection}

This study was carried out during October 2018 to May 2019 on 10 randomly selected mares with history of long barrenness, brought to the VCC of the College in Anand, and from other stud farms of police in Gujarat. After recording anamnesis and rectal palpation findings, the cytological, microbiological and histopathological sampling of the endometrium was carried out. Majority of the mares were barren for exclusion from breeding or failure of conception.

Employing standard aseptic operating procedures, a single-guarded cytobrush (CB) technique, endometrial biopsy (EB) and low volume uterine lavage (LVL) were performed consecutively under xylazine sedation on each animal as described earlier (Mathew et al., 2020). The representative samples harvested by all 3 techniques were processed for cytological and bacteriological evaluation, and biopsies for histopathology. Cloudy or mucoid efflux of LVL was graded as positive and clear efflux as negative.

\section{Cytological and microbiological evaluation}

The samples collected by all the three techniques (CB, EB, LVL) were immediately smeared onto sterile glass slides, air dried, and fixed with methanol. These smears were then stained with Field's stain (A \& B) and were evaluated microscopically at a high power magnification $(40 \mathrm{x}$ or $1000 \mathrm{x})$. Minimum of 10 fields were examined. The smears were evaluated for the presence of epithelial cells, debris, inflammatory cells, bacteria, yeast and total cellularity.

Samples collected for bacteriological isolation from three techniques (CB, EB, LVL) were inoculated onto $5 \%$ sheep blood agar and for fungus isolation on Sabouraud dextrose agar. Cultural characterization were carried out by inoculation further on MacConkey agar, and eosin-methylene blue agar. The bacteria and fungi were identified based on colony characters, morphological and cultural characteristics.

\section{Histopathological examination}

Endometrial samples fixed in $10 \%$ Formalin were processed routinely by paraffin embedding, sectioned at 4-5 $\mu \mathrm{m}$, and stained with hematoxylin and eosin. The biopsies were analyzed for the stage of estrous cycle, the number of fibrotic nests in a 5.5-mm field, and presence of neutrophils, plasma cells, and lymphoid follicles. Mares were classified as having acute endometritis if the inflammatory cells were predominantly neutrophils, chronic endometritis if inflammatory cells were predominantly plasma cells or lymphocytes, and mixed (chronic active) endometritis if 
inflammatory cells within the biopsy included neutrophils, plasma cells, and lymphocytes. Slides were examined for the presence of neutrophils within the endometrial luminal epithelium, stratum compactum and stratum spongiosum. Infiltration of one or more neutrophils (PMNs) per five fields of high magnification $(400 \times)$ was considered as evidence of endometritis (Nielsen, 2005). This method was considered to be the gold standard method for diagnosing endometritis in mares against which other two methods were compared.

\section{Results and Discussion}

\section{Cytological findings}

The smears collected by all the three techniques showed good cellularity. Cytobrush yielded significantly more cells/HPF (57.33 \pm 0.74$)$ than the EB and LVL. The LVL and CB produced more PMNs/HPF $(0.22 \pm 0.24 ; 0.17 \pm 0.18)$ than EB $(0.11 \pm 0.10)$. The proportion of $\mathrm{PMNs} /$ uterine epithelial cells $(1.20 \%)$ was found to be higher in LVL than in smears made from CB $(0.86 \%)$ and EB $(0.48 \%)$. CB collected higher proportion of PMNs than EB. None of the smears revealed severe inflammation (> 5 PMNs/HPF). Regardless of the method used for sample collection, endometritis was diagnosed more frequently if smears were evaluated according to criterion II $(\geq 1 \%$ PMN/300 epithelial cells), i.e., in 30 (3/10) \% of the mares by EB and $\mathrm{CB}$ samples and 40 (4/10) \% by LVL as compared to criterion I ( $\geq 1 \mathrm{PMN} / \mathrm{HPF}$ ) by which none was diagnosed positive. The $\mathrm{CB}$ harvested significantly more fragmented cells than EB and LVL. Distorted cells were significantly more frequent in smears made from LVL than in those collected by $\mathrm{EB}$ and $\mathrm{CB}$. The $\mathrm{EB}$ yielded significantly more intact cells $(90 \%)$ than CB (60\%) and LVL (42\%). Distorted cells were significantly more frequent in smears made from LVL than in those collected by EB and $\mathrm{CB}$. The $\mathrm{CB}$ harvested significantly more fragmented cells than EB and LVL.

\section{Microbiological findings}

In this study, positive growth was obtained from EB in $7(70 \%)$ mares, from CB in $6(60$ $\%)$ and from LVL fluid in $8(80 \%)$ mare. In 3 of $10(30 \%)$ cytological smears obtained from $\mathrm{EB}$ and $\mathrm{CB}$ and in 4 of $10(40 \%)$ cytological smears obtained from LVL, the percentage of PMNs was $\geq 1$. In EB, CB and LVL, all the cytological positive mares also had positive cultures, whereas $30 \%$ positive cultures from $\mathrm{CB}$ and $40 \%$ positive cultures from EB and LVL had negative cytology. The most common pathogenic bacteria cultured from EB was $\beta$-hemolytic Streptococci either alone $(30 \%)$ or in combination with other microorganism, and from $\mathrm{CB}, \beta$-hemolytic Streptococci and E. coli were isolated in equal proportions, whereas, in LVL, E. coli was isolated most frequently either alone (40\%) or in combination with other micro-organisms $(10 \%)$ and $\beta$-hemolytic Streptococci was the second most frequently isolated organism (20 $\%$ alone; $10 \%$ in combination with other organisms). This was in agreement with the study conducted by LeBlanc et al., (2007).

The different types of cells and microbes obtained from 3 types of samples are depicted in plates 1 to 4 . The association of endometrial cytology and microbiology evaluated is presented in Table 1 and Figure 1. We did not find much difference in the number of positive cultures between $\mathrm{CB}, \mathrm{EB}$ and LVL or in positive cytology findings between the three methods. In a study conducted by Overbeck et al., (2011), bacteriological findings were not associated with positive cytology. Similar observations were reported by Riddle et al., (2007) and Bindslev et al., (2008). LeBlanc et al., (2007) 
has shown in hysteroscopic examinations that $E$. coli can occur in local areas which can explain some of the failures to diagnose $E$. coli.

In the present study, E. coli was present in $33.33 \%$ samples with $\mathrm{PMNs}<1 \%$, while $\beta$ haemolytic Streptococcus was isolated in $26.67 \%$ samples with PMNs $>1 \%$, but not the vis-a-vis (Table 2. Fig. 2). The results clearly show the importance of performing both bacteriological and cytological examinations of the endometrium, as it is not sufficient to rely on either method alone, a suggestion that has been raised by several studies in the past (Nielsen, 2005; Riddle et al., 2005). This is particularly true in relation to $E$. coli infections, where a diagnosis based on positive cytology is likely to result in a considerable number of false negative samples.

\section{Histopathological examination of the endometrial biopsies}

In the present study, a total of $6(60 \%)$ mares had positive histopathology for endometritis considering infiltration of the endometrial luminal epithelium and stratum compactum by one or more PMNs per five fields of high magnification $(400 \mathrm{x})$. The inflammatory changes could be evidenced from the histopathological slides prepared from the endometrial biopsy samples (plate 5). It shows the presence of PMN cell infiltration in the stratum compactum (A) as well as varying degrees of fibrotic changes, from distension of uterine glands with inflammatory cell infiltration and fibrosis (B, $\mathrm{C}, \mathrm{D})$, to the presence of fibrotic nests of different size $(\mathrm{E}, \mathrm{F})$.

Histopathological examination of an endometrial biopsy, as shown in this study, is the most reliable diagnostic test for endometritis and uterine infection. The histological examination allows detection of inflammatory response caused by uterine infectious agents and evaluation of chronic degenerative disorders of the endometrium in the same procedure (Ricketts, 1999). A practical disadvantage of this method is however, the duration between sampling and laboratory results. This time may not be available for a mare presented in estrus during the breeding season. In comparison, bacteriological results can be cultured overnight.

The results of cytological examinations were compared for the presence of infiltration by PMNs of the luminal epithelium and stratum compactum because histological examination of the endometrium is generally used as the reference standard for diagnosis of endometritis (Nielsen, 2005; LeBlanc et al., 2007; Nielsen et al., 2012; Overbeck et al., 2011; Overbeck et al., 2013; Buczkowska et al., 2014). However, interpreting these results we should remember that no diagnostic test is fully accurate. For example, some observations indicate that one biopsy sample is not fully representative of the whole uterus (Fiala, 2010), but in contrast to these observations Overbeck et al., (2013) found no significant differences in PMN numbers in biopsy material collected post-mortem from different locations of the equine uterus. Additionally, Nielsen et al., (2012) showed a strong correlation between the presence of PMNs in endometrial tissue determined by histology and pregnancy results on day 70, but there was no correlation between pregnancy detected on day 70 and results of cytological examination. Petersen et al., (2009) reported that bacteria residing deeper in the endometrium are not recovered by surface sampling techniques, and hence biopsy in more reliable and confirmatory of endometritis. 
Table.1 Association of endometrial culture and cytology (PMNs+/-) for samples collected by endometrial biopsy, cytobrush and low volume uterine lavage in barren mares

\begin{tabular}{|c|c|c|c|c|c|c|}
\hline \multirow[t]{2}{*}{ Bacterial isolates } & \multicolumn{6}{|c|}{ Cytology positive $(\mathrm{C}+)$ / negative $(\mathrm{C}-)$} \\
\hline & EBC+ & EBC- & $\mathrm{CBC}+$ & CBC- & LVLC+ & LVLC- \\
\hline Sterile & 0 & 3 & 0 & 4 & 0 & 2 \\
\hline $\begin{array}{l}\beta \text { hemolytic } \\
\text { Streptococcus }\end{array}$ & 3 & 0 & 3 & 0 & 2 & 0 \\
\hline E. coli & 0 & 3 & 0 & 3 & 0 & 4 \\
\hline $\begin{array}{l}\text { E. coli }+\beta \text { hemolytic } \\
\text { Streptococcus }\end{array}$ & 0 & 0 & 0 & 0 & 1 & 0 \\
\hline $\begin{array}{l}\beta \text { hemolytic } \\
\text { Streptococcus+Yeast }\end{array}$ & 0 & 1 & 0 & 0 & 0 & 0 \\
\hline $\begin{array}{l}\text { Staphylococcus spp. }+ \\
\text { Psedomonas spp. }\end{array}$ & 0 & 0 & 0 & 0 & 1 & 0 \\
\hline Total out of 10 & 3 & 7 & 3 & 7 & 4 & 6 \\
\hline
\end{tabular}

EBC+/- ; Endometrial biopsy (cytology positive / cytology negative)

$\mathrm{CBC}+/-$; Cytobrush (cytology positive / cytology negative)

LVLC+/- ; Low-volume uterine lavage (cytology positive / cytology negative)

Table.2 Presence of PMNs during infection with E. coli versus other agents: Comparison of the results obtained by the different collection techniques

\begin{tabular}{|l|c|c|c|c|c|c|c|c|c|}
\hline \multirow{2}{*}{$\begin{array}{c}\text { Bacterial } \\
\text { Isolates }\end{array}$} & \multicolumn{3}{|c|}{ Endometrial biopsy } & \multicolumn{3}{c|}{ Cytobrush } & \multicolumn{3}{c|}{ LVL } \\
\hline Agent & Cyto (+) & Cyto (-) & Total & Cyto (+) & Cyto (-) & Total & Cyto (+) & Cyto (-) & Total \\
\hline E. coli & 0 & No. (\%) & No. (\%) & No. (\%) & No. $(\%)$ & No. (\%) & No. (\%) & No. (\%) & No. (\%) \\
\hline Others & $3(30)$ & $1(10)$ & $3(30)$ & 0 & $3(30)$ & $3(30)$ & 0 & $4(40)$ & $4(40)$ \\
\hline Total & $3(30)$ & $4(40)$ & $7(70)$ & $3(30)$ & 0 & $3(30)$ & $4(40)$ & 0 & $4(40)$ \\
\hline
\end{tabular}

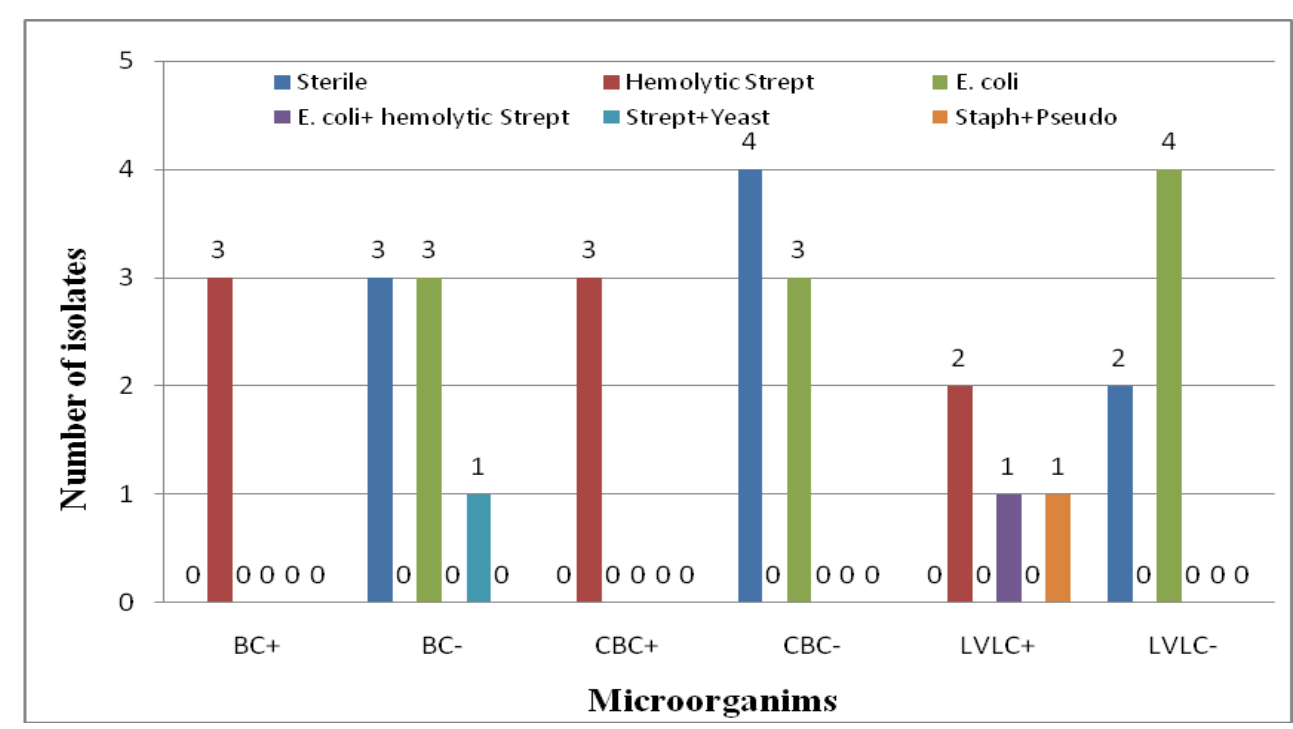

Fig.1 Comparison of endometrial culture and endometrial cytology (PMNs+/-) using different sample collection techniques (EB, CB, LVL) 


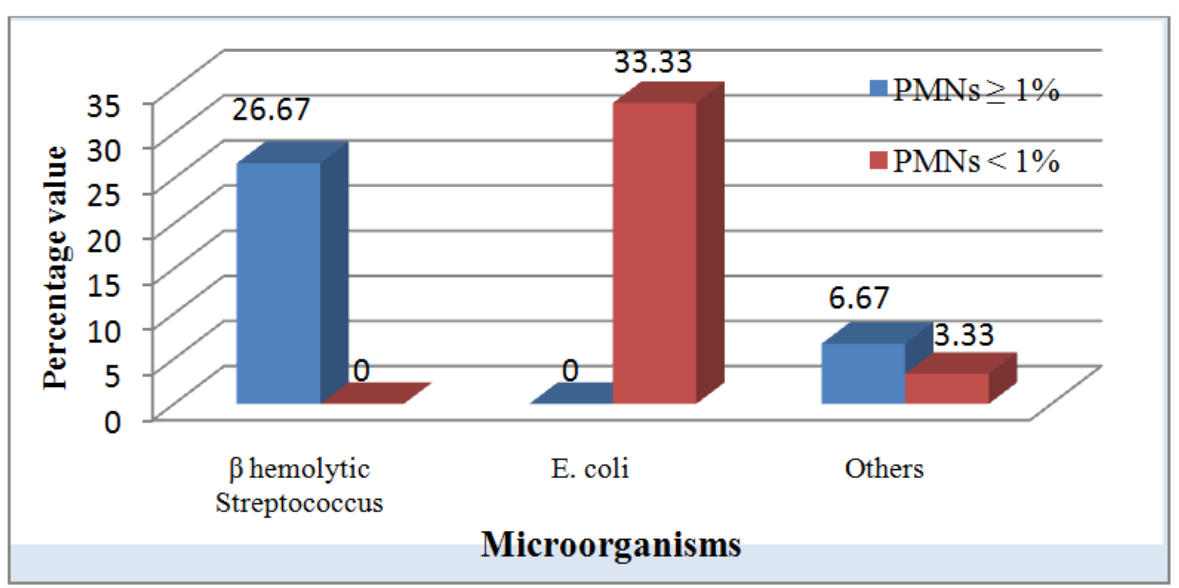

Fig.2 Isolated bacteria and their evidence of inflammation in cytology obtained from endometrial biopsy, cytobrush and low-volume uterine flush
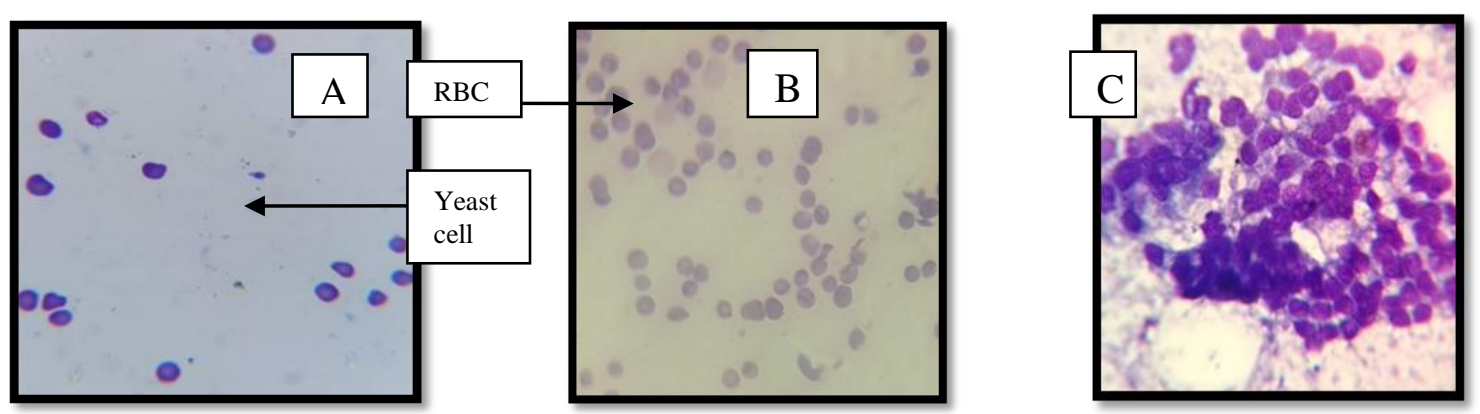

Plate.1 Cytology smear prepared from endometrial biopsy showing presence of yeast cell (A), red blood cells (B) and good cellularity (C)

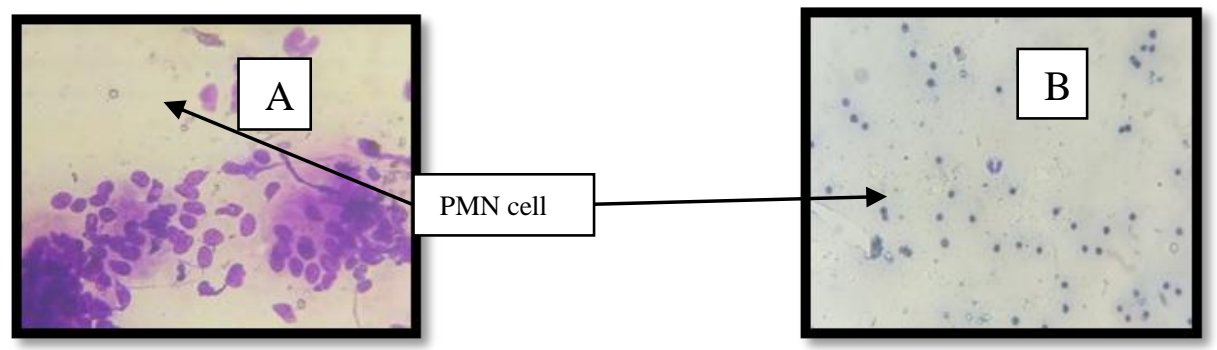

Plate.2 Cytology smears prepared from cytobrush showing clear background with presence of PMN cells, 1000x (A) and 400x (B)
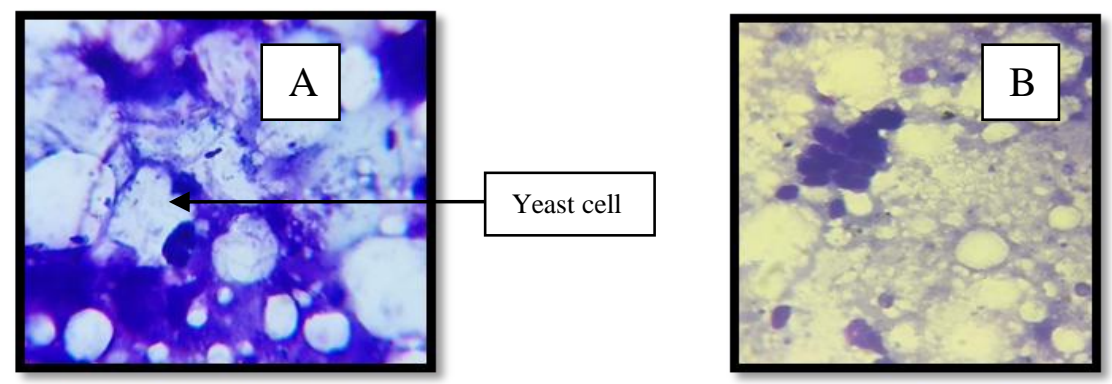

Plate.3 Cytology smears prepared from LVL fluid showing presence of yeast cell (A) and debris 

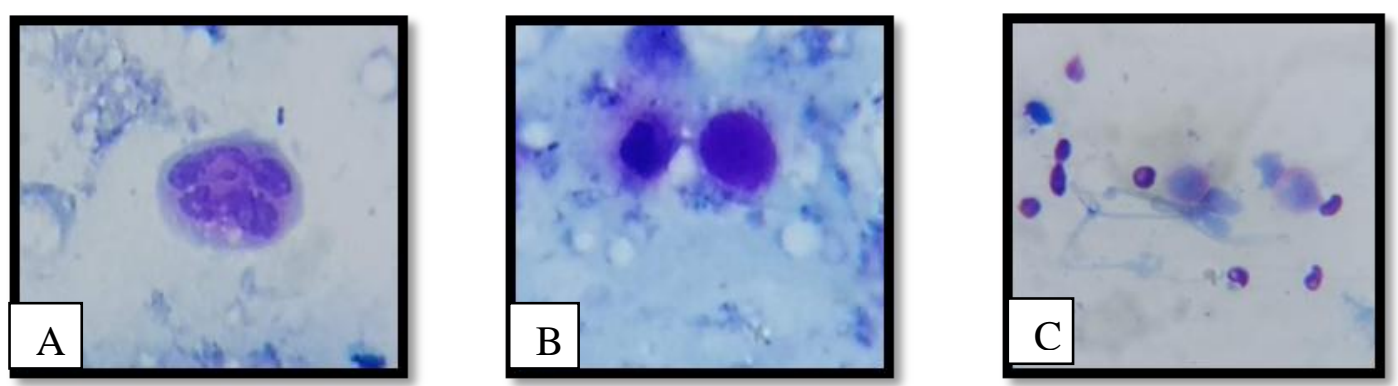

Plate.4 Cytology smears prepared from CB showing PMN cell (A) and lymphocyte (B) and EB smear $(\mathrm{C})$ showing the presence of monocytes
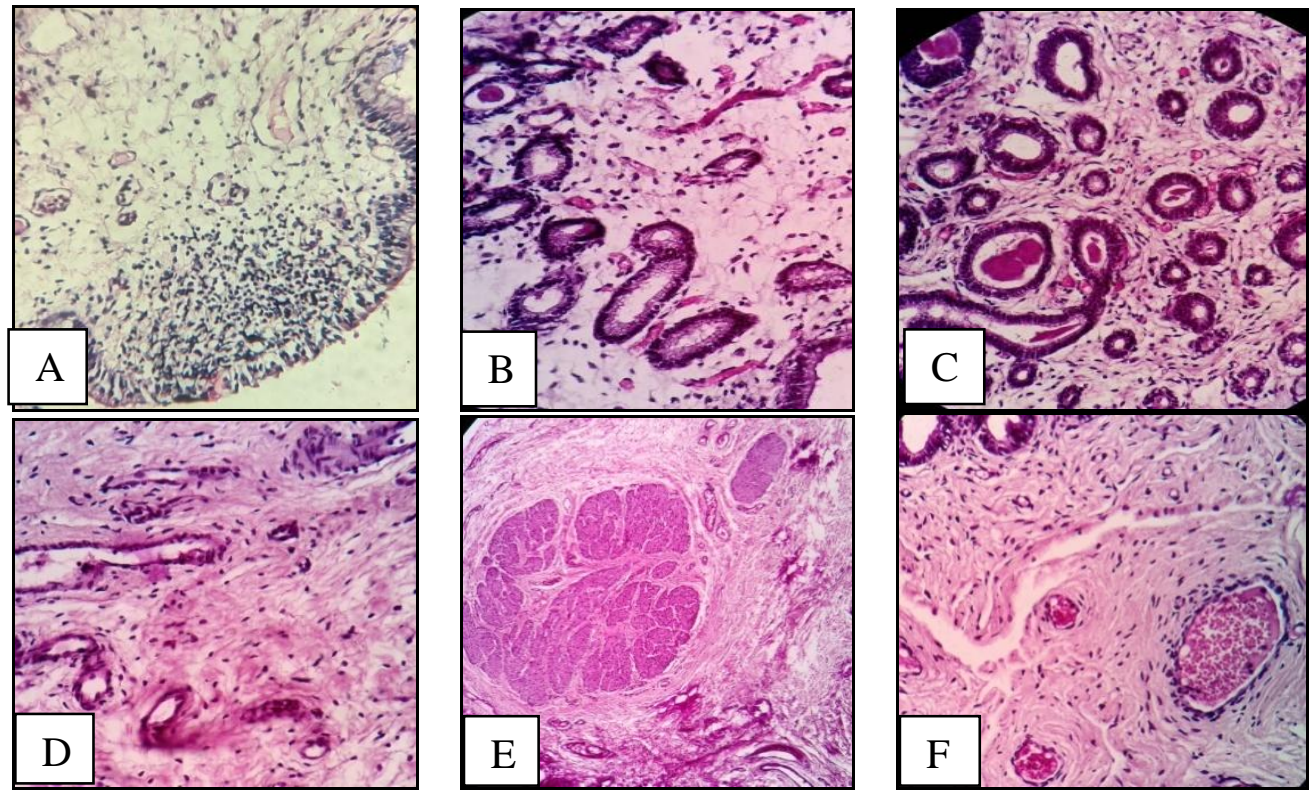

Plate.5 Histology sections of endometrial biopsies showing, acute and chronic lesions.

Infiltration of stratum compactum by PMNs (A), dialated uterine glands with PMN infiltration (B), mild periglandular fibrosis (C), glandular atrophy and fibrosis (D), fibrotic nest (E), severe periglandular fibrosis $(\mathrm{F})$

These results support the hypothesis that the presence of PMNs in endometrial tissue is currently the most reliable method for diagnosis of endometritis and can be used as the "best standard" in studies focused on estimating the suitability of methods in the diagnosis of subclinical endometritis in horses. A total of 6 out of $10(60 \%)$ mares had positive histopathology. No statistical differences in the number of positive cultures were obtained between different techniques. In EB, CB and LVL, all the cytological positive mares also had positive cultures. Whereas, $30 \%$ positive cultures from $\mathrm{CB}$ and $40 \%$ positive cultures from EB and LVL had negative cytology. The most common bacteria isolated were $\beta$-hemolytic Streptococci and $E$. coli alone or as mixed infection of both the organisms. Positive correlation was observed between isolation of $\beta$-hemolytic Streptococci with positive cytology, but not for $E$. coli isolation.

\section{Acknowledgement}

We thank the authorities of AAU, Anand and Dean of Veterinary College for the facilities provided and the animal owners for their support and cooperation while collecting uterine samples from their animals. 


\section{References}

Beehan, D.P., Wolfsdorf, K., Elam, J., Krekeler, N., Paccamonti, D. and Lyle, S.K. 2015. The evaluation of biofilm-forming potential of Escherichia coli collected from the equine female reproductive tract. J. Equine Vet. Sci., 35, 935-939.

Bindslev, M.M., Villumsen, H., Petersen, M.M., Nielsen, J.M., Bogh, I.B. and Bojesen, A.M. 2008. Genetic diversity of $S$. equi ssp. zooepidemicus and E. coli isolated from the reproductive tract of the mare. Proc. Intl. Congress on Anim. Reprod. (Supple 3), Budapest, Hungary.

Buczkowska, J., Kozdrowski, R., Nowak, M., Ras, A., Staroniewicz, Z. and Siemieniuch, M.J. 2014. Comparison of the biopsy and cytobrush techniques for diagnosis of subclinical endometritis in mares. Reprod. Biol. Endocrinol., 12(1), 27.

Ferris, R.A., Wittstock, S.M., McCue, P.M. and Borlee, B.R. 2014. Evaluation of biofilms in Gram-negative bacteria isolated from the equine uterus. J. Equine Vet. Sci., 34, 121.

Fiala, S.M. 2010. Endometrial fibrotic changes. Is one biopsy enough to diagnose degenerative changes? Anim. Reprod. Sci., 121, 89-90.

LeBlanc, M.M. 2010. Advances in the diagnosis and treatment of chronic infectious and postmating-induced endometritis in the mare. Reprod. Dom. Anim., 45, 21-27.

LeBlanc, M.M. and Causey, R.C. 2009. Clinical and subclinical endometritis in the mare: both threats to fertility. Reprod. Dom. Anim., 44, $10-22$.

LeBlanc, M.M., Magsig, J. and Stromberg, A.J. 2007. Use of a low-volume uterine flush for diagnosing endometritis in chronically infertile mares. Theriogenology, 68(3), 403412.

Mathew Agnus, Patel, D.M. and Hadiya, K.K. (2020). Sensitivity, specificity and predictive values of cytological and microbiological findings of endometrial biopsy, cytobrush and low volume uterine lavage in relation to endometrial histology in barren mares. Indian J. Vet. Sci. \& Biotech., 15(4): 48-53.

Nielsen, J.M. 2005. Endometritis in the mare: A diagnostic study comparing cultures from swab and biopsy. Theriogenology, 64, 510518.

Nielsen, J.M., Nielsen, F.H. and Petersen, M.R. 2012. Diagnosis of equine endometritis Microbiology, cytology and histology of endometrial biopsies and the correlation to fertility. Pferdeheilkunde, 28(1), 8-13.

Overbeck, W., Jäger, K., Schoon, H.A. and Witte, T.S. 2013. Comparison of cytological and histological examinations in different locations of the equine uterus - an in vitro study. Theriogenology, 79(9), 1262-1268.

Overbeck, W., Witte, T.S. and Heuwieser, W. 2011. Comparison of three diagnostic methods to identify subclinical endometritis in mares. Theriogenology, 75(7), 1311-1318.

Petersen, M.R., Nielsen, J.M., Lehn-Jensen, H. and Bojesen, A.M. 2009. Streptococcus equi subsp. Zooepidemicus resides deep in the chronically infected endometrium of mares. Clin. Theriogenol., 1, 393-409.

Ricketts, S.W. 1999. The treatment of equine endometritis in stud farm practice. Pferdeheilkunde, 15(6), 588-593.

Riddle, W.T., LeBlanc, M.M., Pierce, S.W. and Stromberg, A.J. 2005. Relationships between pregnancy rates, uterine cytology, and culture results in a Thoroughbred practice in Central Kentucky. In Proc. $51^{\text {st }}$ Annual Conv. of Am. Assoc. Equine Pract., Seattle, Washington, USA, 3-7 December, p. 198-201.

Riddle, W.T., LeBlanc, M.M. and Stromberg, A.J. 2007. Relationships between uterine culture, cytology and pregnancy rates in a Thoroughbred practice. Theriogenology, 68(3), 395-402.

\section{How to cite this article:}

Agnus Mathew, D. M. Patel, K. K. Hadiya, B. B. Bhanderi and Ghodasara. D. J. 2020. Association of Cytology, Microbiology and Histopathology of Cytobrush, Uterine Lavage and Endometrial Biopsy Samples from Mares with Endometritis. Int.J.Curr.Microbiol.App.Sci. 9(06): 555-562. doi: https://doi.org/10.20546/ijcmas.2020.906.072 